\title{
The Relationship between Heart Rate Variability and Adiposity Differs for Central and Overall Adiposity
}

\author{
B. Gwen Windham, ${ }^{1}$ Stefano Fumagalli, ${ }^{2}$ Alessandro Ble, ${ }^{3}$ John J. Sollers, ${ }^{4}$ Julian F. Thayer, ${ }^{5}$ \\ Samar S. Najjar, ${ }^{3,6}$ Michael E. Griswold, ${ }^{7}$ and Luigi Ferrucci ${ }^{3}$ \\ ${ }^{1}$ Division of Geriatrics, Department of Medicine, University of Mississippi Medical Center, Jackson, MS 39216, USA \\ ${ }^{2}$ Unit of Geriatrics, Department of Critical Care Medicine and Surgery, University of Florence and AOU Careggi, \\ 50134 Florence, Italy \\ ${ }^{3}$ Clinical Research Branch, National Institute on Aging, National Institutes of Health, Baltimore, MD 21225-1290, USA \\ ${ }^{4}$ Department of Psychological Medicine, Faculty of Medical \& Health Science, The University of Auckland, \\ Auckland 1142, New Zealand \\ ${ }^{5}$ The Ohio State University, Columbus, OH 43210, USA \\ ${ }^{6}$ Laboratory of Cardiovascular Science, Department of Biostatistics, Johns Hopkins Bloomberg School of Public Health, \\ Baltimore, MD 21205, USA \\ ${ }^{7}$ Center of Biostatistics and Bioinformatics, University of Mississippi Medical Center, Jackson, MS 39216, USA
}

Correspondence should be addressed to Julian F. Thayer, thayer.39@osu.edu

Received 15 December 2011; Revised 23 February 2012; Accepted 23 February 2012

Academic Editor: Carl J. Lavie

Copyright ( 2012 B. Gwen Windham et al. This is an open access article distributed under the Creative Commons Attribution License, which permits unrestricted use, distribution, and reproduction in any medium, provided the original work is properly cited.

While frank obesity is associated with reduced HRV, indicative of poorer autonomic nervous system (ANS) function, the association between body mass index (BMI) and HRV is less clear. We hypothesized that effects of adiposity on ANS are mostly mediated by visceral fat and less by subcutaneous fat; therefore, centrally distributed adipose tissue, that is, waist circumference (WC), should be more strongly associated with HRV than overall adiposity (BMI). To examine this hypothesis, we used data collected in a subset of the Baltimore Longitudinal Study of Aging to compare strength of association between HRV and WC to that of HRV and BMI. Time domain HRV variables SDNN (standard deviation of successive differences in normal-to-normal $(\mathrm{N}-\mathrm{N})$ intervals) and RMSSD (root mean square of successive differences in N-N intervals) were calculated from 24-hour Holter recordings in 159 participants (29-96 years). Increasing WC was associated with decreasing SDNN and RMSSD in younger but not older participants $(P$ value for WC-by-age interaction $=0.003)$. BMI was not associated with either SDNN or RMSSD at any age. In conclusion, central adiposity may contribute to sympathetic and parasympathetic ANS declines early in life.

\section{Introduction}

The autonomic nervous system (ANS) directs voluntary and involuntary physiologic processes such as digestion, blood pressure, hormonal regulation, energy metabolism, and heart rate and is therefore considered an important regulator of homeostasis [1-3]. Heart rate variability (HRV) is widely considered a standard noninvasive method for assessing ANS function, due to ANS regulation of heart rate in a continuous, beat-to-beat manner [4], with lower HRV generally considered an indicator of poorer autonomic function. Lower HRV or autonomic dysfunction has been associated with several conditions of significant public health concern, including diabetes and diseases of the cardiovascular system [5-11].

Obesity, an important risk factor for diabetes and cardiovascular disease, is associated with dysregulation of autonomic function. It has been suggested that ANS dysfunction is an important mediator in the development of obesityassociated disease and insulin resistance although the nature of the link between adiposity and insulin sensitivity is still unclear [12-15]. Studies of the relationship between body 
mass index (BMI) and HRV have reported conflicting results with stronger associations observed in younger populations [16-19]. Understanding the mechanism that connects obesity and ANS function is important because of the increasing obesity prevalence documented among men and women of all ages [20]. One explanation for controversial data on the associations between BMI and HRV is that the distribution of body fat may be more important than a measure of overall obesity, such as BMI, due to the metabolic activity of visceral or abdominal adipose tissue [21-23]. This could be especially problematic in older persons for whom BMI might be a poorer indicator of adiposity [24]. We hypothesized that central (abdominal) adiposity, not overall adiposity, accounts for obesity-related autonomic dysfunction. To examine this hypothesis, we compared the strength of association between HRV and WC to that of HRV and BMI, anticipating stronger, negative associations between HRV and WC compared to that of HRV and BMI. Furthermore, since obesity tends to be a stronger risk factor for cardiovascular morbidity and mortality in young and middle-aged persons than in older persons, we examined whether WC and BMI relationships with ANS differ by age, independent of specific health conditions known to impact HRV.

\section{Methods}

2.1. Study Population. The Baltimore Longitudinal Study of Aging (BLSA) is an observational study sponsored by the National Institutes of Health and the National Institute on Aging (NIA) that began in 1958 to study normative aging in a volunteer cohort of healthy persons 18 years of age and older at study entry [25]. Most BLSA participants reside in the eastern USA, predominantly the Baltimore-Washington DC area, and visit the NIA Advanced Studies in Translational Research on Aging Unit every one to four years depending on their age. In 2004, 24-hour ambulatory electrocardiograms were implemented in the BLSA to assess HRV.

The first two hundred fourteen participants with Holter data analyzed consecutively in the BLSA were evaluated. These participants underwent a two-to-three day assessment between March 2004 and March 2006, which included a battery of tests including a standardized medical examination and interview, sociodemographic interview, anthropometric assessment, fasting blood tests, and an oral glucose tolerance test (OGTT) using a standard 75 gram glucose load. All participants signed informed consent. This study complied with the ethical rules for human experimentation as stated in the Declaration of Helsinki and was approved by the MedStar Research Institute institutional review board.

2.2. Outcomes: HRV Assessment. HRV was assessed from ambulatory 24-hour Holter monitors that use digitalized technology with compact flashcards and HRV software (DelMar Reynolds Lifecard CF, 1024 samples per second, Impresario software). An initial analysis was planned for the first 200 recordings processed, and 214 had been completed at the time of this analysis. All recordings were visually reviewed and manually edited in a beat-by-beat analysis using a standardized protocol to identify artifact and nonsinus beats.

Exclusion criteria for HRV analysis on Holter data were (1) implanted pacemakers, (2) transient or persistent nonsinus rhythm during the recording, such as atrial fibrillation, and (3) wandering atrial pacemakers (WAPs). Although WAP is generally considered a benign condition, it may produce invalid readings and constitutes a typical exclusion [26-28]. HRV measures can be affected by frequent premature beats, artifact, and total hours of recordings. Thus, HRV data were excluded when recordings were less than 18 hours, more than $10 \%$ of beats were premature, or artifact time exceeded $5 \%$ of recorded time [4].

This analysis focused on standard time domain HRV measurements, SDNN (standard deviation of successive differences in normal-to-normal (N-N) intervals), and RMSSD (root mean square of successive differences between $\mathrm{N}$ $\mathrm{N}$ intervals). SDNN represents a measure of overall ANS function (sympathetic and some parasympathetic function) while RMSSD represents a measure of parasympathetic function [4]. Time domain measures have been suggested for ambulatory studies as they are less susceptible to respiratory and movement artifacts. Because HRV can be affected by activity and sleep, SDNN and RMSSD were obtained separately for day (6:00 AM-10:30 PM unless otherwise reported in diary and was of similar hours) and night (1:00 AM-5:00 AM). Nighttime hours were also selected to provide a constant length of time for HRV analysis in which participants were all sleeping.

The autonomic nervous system contributes to the circadian patterns of HRV with sympathetic nerve discharges associated with activity, such as during daytime wakefulness [29]. The circadian patterns of parasympathetic activity also demonstrate a peak which occurs at night and plateaus during the day [30]. Because of this, the HRV outcomes of interest will focus on daytime SDNN, to describe overall function, sympathetic more so than parasympathetic function, and nighttime RMSSD, to describe parasympathetic function.

2.3. Predictors. Height, weight, and waist circumference, (WC, centimeter, one inch above the anterior superior iliac crests) were measured with participants disrobed or in light clothes and barefoot. Height was measured using a stadiometer. WC and height were measured three times and averaged. BMI was calculated as weight in kilograms/height in meters $^{2}$ as a measure of overall adiposity whereas WC was considered a measure of central or visceral adiposity. This was based on findings from the Rancho Bernardo Heart and Chronic Disease Study, which showed WC to be strongly correlated with most measures of central obesity in men and women aged 65-96 years [31].

2.4. Covariates. Conditions known to be associated with HRV and body composition were assessed as covariates, including glucose intolerance, hypertension, and physical activity $[10,12,32]$. Glucose metabolism was assessed 
with a 2-hour oral glucose tolerance test (OGTT). Blood pressure was taken using a manual sphygmomanometer with the participant resting supine for 5 minutes in a quiet, climate-controlled room with low lighting, using the first and fifth Korotkoff sounds. The second and third systolic blood pressure (SBP) and diastolic blood pressure (DBP) measurements were averaged. Hypertension was defined as use of antihypertensive medication, self-reported diagnosis confirmed with medical record review, or SBP $>140 \mathrm{~mm} \mathrm{Hg}$ or DBP $>90 \mathrm{~mm} \mathrm{Hg}$ during the physical examination. Diabetes was defined as use of hypoglycemic medications, selfreported diagnosis of diabetes confirmed by medical records, fasting glucose of $126 \mathrm{mg} / \mathrm{dL}$ or greater, or a 2-hour OGTT value $\geq 200 \mathrm{mg} / \mathrm{dL}$ [33]. Angina and myocardial infarction were assessed by self-report, review of medical records, and by major Q-waves on resting electrocardiograms using standard Minnesota Coding system criteria. Due to the infrequent occurrence of diabetes, angina, and myocardial infarction, analyses were conducted including and then excluding participants with these conditions. Self-reported physical activity was categorized by metabolic equivalents per kg per week [34]. Age and race were obtained from a standardized interview. Race was categorized as black (self-reported race as "black" or "African American") and nonblack.

2.5. Statistical Analysis. Simple comparisons of continuous variables by sex were performed with nonparametric Wilcoxon tests and categorical variables with chi-square tests. The correlation between continuous variables was assessed using Spearman's correlation coefficient. Because BMI and WC have different units, we used standardized coefficients of each to assess whether one was associated more with the HRV outcomes than the other. Akaike's Information Criterion (AIC) was used to compare models (WC versus BMI), with lower AIC indicating a better fitted model. To account for nonnormal distributions of HRV, standard generalized linear models with gamma distribution and log link were used to assess relationships between adiposity measures (BMI and WC) and HRV (nighttime RMSSD and daytime SDNN). To determine whether these relationships differ by age, age-byBMI and age-by-WC interaction terms were included. All models were adjusted for age, sex, race, physical activity, and hyperglycemia using the OGTT 2-hour glucose. Previously published studies suggest that HRV may differ across age by sex and race, and some studies suggest that glucose metabolism differs by sex $[35,36]$. Thus, we also [34] evaluated these interactions by including age-by-sex, age-byrace, and sex-by-OGTT glucose terms. Significance level was defined as $P<.05$.

Relationships were reported as relative ratios (RR), to describe the multiplicative increases and decreases in HRV associated with a unit change in the explanatory variable, similar to a relative risk for binary data. For example, an RR of 1.2 for male sex suggests a $20 \%$ higher HRV in men than in women.

Lastly, because of the potential for beta blockers to improve HRV [37], we repeated all analyses after excluding participants on beta blockers. All analyses were conducted using SAS 9.1 statistical software (SAS Institute Inc., Cary, $\mathrm{NC}$ ).

\section{Results}

Of 214 participants with Holter data, we excluded two participants with recordings less than 18 hours, one with nonsinus rhythm who also had a pacemaker, and 33 with wandering atrial pacemakers, leaving 178 with HRV data. Among these, nine were excluded from the OGTT (two were taking insulin, six used corticosteroids in the previous three months, and one refused). Of the 169 remaining participants, three were missing data on WC and seven were missing OGTT glucose due to difficult intravenous access, leaving 159 participants for the current analysis. Of these, one participant lacked a nighttime assessment. Only 15 participants $(8 \%)$ were diabetic and $8(4.5 \%)$ had angina or myocardial infarction; 116 (65\%) were hypertensive. Sixtyseven participants $(37 \%)$ were 70 years or older.

Characteristics of the 178 participants with valid HRV data are presented in Table 1. Figure 1 shows histograms of the HRV variables. The similarity between the lines representing the gamma distribution and the kernel density smoother of the actual data suggests a good fit of the gamma model.

WC and BMI were significantly correlated $(r=0.73$, $P<.0001)$. Despite this high correlation, a wide range of WC was observed for any given BMI, suggesting that WC describes potentially different information than BMI in this population.

3.1. RMSSD (Parasympathetic ANS Function). The relationship between RMSSD and WC differed by age, with RMSSD decreasing as WC increased in younger but not older participants $(P$ for interaction $=0.008)$ (Table 2 and Figure 2 , left panel). Figure 2 illustrates a comparison of the expected nighttime RMSSD, based on model results, for an average participant at two different ages: 45 years and 85 years if the standardized WC increased. With increasing WC, the RMSSD decreases in the 45-year-old but not in the 85-yearold participant. In contrast, neither the main effect of BMI nor the BMI-by-age interaction was statistically significant, suggesting that BMI was not associated with RMSSD at young or old ages $(P=0.403)$ (Figure 2, right panels). Furthermore, the AIC for the RMSSD models suggested that WC provided a better fitted model than did BMI (AIC $=819$ versus 842).

3.2. SDNN (Overall ANS Function). Increasing WC was also associated with decreasing SDNN in younger but not older participants $(P$ for interaction $=0.003)($ Figure 2 , Table 2$)$. There was no statistically significant association between BMI and SDNN (Figure 2, Table 2). In addition, the model fit for WC was superior to the model fit for BMI (AIC $=953$ versus 982).

When participants with coronary artery disease or diabetes or those taking beta blockers were excluded, we found 

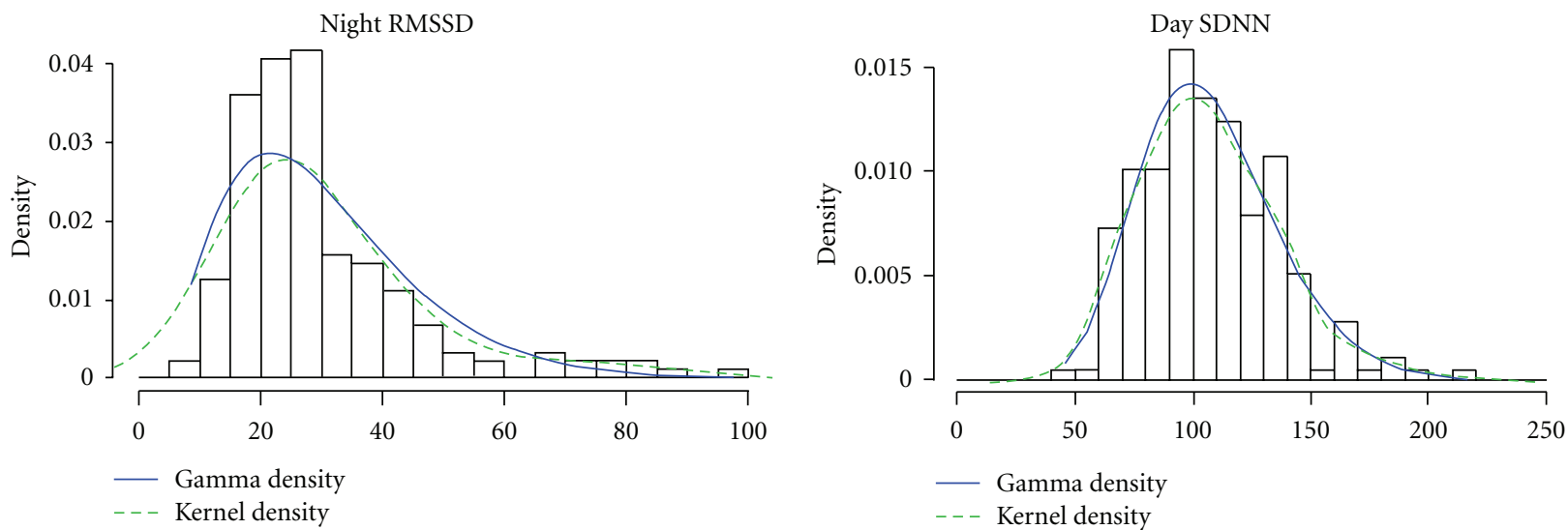

FIGURE 1: Histograms of heart rate variability variables with a smooth kernel density estimate (dashed line) and gamma distribution (solid line). RMSSD: root mean square of successive differences; SDNN: standard deviation of N-N intervals.

TABLE 1: Sample characteristics stratified by sex.

\begin{tabular}{lccc}
\hline & Women $(n=90)$ & Men $(n=88)$ & $P$ value \\
& \multicolumn{2}{c}{ Mean $(\mathrm{SD})$ or $N(\%)$} & 0.096 \\
\hline SDNN $\left(\mathrm{ms}^{2}\right)$ & $103(25.6)$ & $110(30.0)$ & 0.815 \\
RMSSD $\left(\mathrm{ms}^{2}\right)$ & $30(14.6)$ & $30(16.7)$ & 0.198 \\
Age years & $64(14.3)$ & $66(11.8)$ & 0.107 \\
Systolic blood pressure $(\mathrm{mm} \mathrm{Hg})$ & $119(16.6)$ & $123(14.2)$ & 0.226 \\
Body mass index $\left(\mathrm{kg} / \mathrm{m}^{2}\right)$ & $27(4.9)$ & $27(3.9)$ & $<0.001$ \\
Waist circumference $(\mathrm{cm})$ & $88(14.0)$ & $98(12.9)$ & 0.026 \\
Fasting glucose $(\mathrm{mg} / \mathrm{dL})$ & $93(13.1)$ & $98(17.7)$ & 0.067 \\
OGTT 2-hour glucose $(\mathrm{mg} / \mathrm{dL})$ & $118(43.5)$ & $133(54.7)$ & 0.681 \\
Black race & $26(29 \%)$ & $24(26 \%)$ & 0.371 \\
Antihypertensive use & $40(44 \%)$ & $46(52 \%)$ & 0.317 \\
Beta blocker use & $16(18 \%)$ & $21(24 \%)$ & 0.353 \\
Hypertension & $50(56 \%)$ & $56(63 \%)$ & \\
\hline
\end{tabular}

${ }^{\dagger}$ Self-reported race/ethnicity $(N)$ included: Caucasian (118), black or African American (50), Asian or Pacific islanders (4), American Indian or native Alaskan (1), Hispanic (2), Chinese (3), and other or unknown (1).

ms:milliseconds; OGTT: oral glucose tolerance test; RMSSD: root mean square of successive differences in normal-to-normal intervals; SDNN: standard deviation of normal-to-normal intervals.

similar results for both SDNN and RMSSD. Specifically, the parameter estimates remained similar, and statistical significance was unchanged despite a smaller sample (data not shown).

\section{Discussion}

To our knowledge, this is the first study to assess the roles of overall and abdominal adiposity on ANS function in young and old persons. The findings showed that central adiposity, measured by waist circumference, was associated with poorer ANS function at younger but not older ages. This relationship was observed for a measure of overall ANS function that includes sympathetic and parasympathetic activity and for a measure of primarily parasympathetic activity. Overall adiposity, measured using BMI, was not associated with either measure of ANS function. Thus, abdominal adiposity, as opposed to overall adiposity tissue, could adversely affect ANS function at younger ages with potentially unfavorable effects in later life.

Our results are in accordance with a small study by Christou et al. [38], which reported higher abdominal-toperipheral body fat distributions measured by DXA were strongly correlated with lower sympathetic and parasympathetic function in young (mean age $25 \pm 1$ year) and old (mean age $65 \pm 1$ year) healthy men. Other studies have also shown that body fat distribution accounts for obesityassociated risk more so than total fat, with visceral fat carrying the greatest risk for insulin resistance, hyperglycemia, hypertriglyceridemia, cardiovascular disease, metabolic syndrome, and mortality [22, 23, 39-41]. Our findings suggest that abdominal adiposity may also contribute to poorer sympathetic and parasympathetic function as well. 

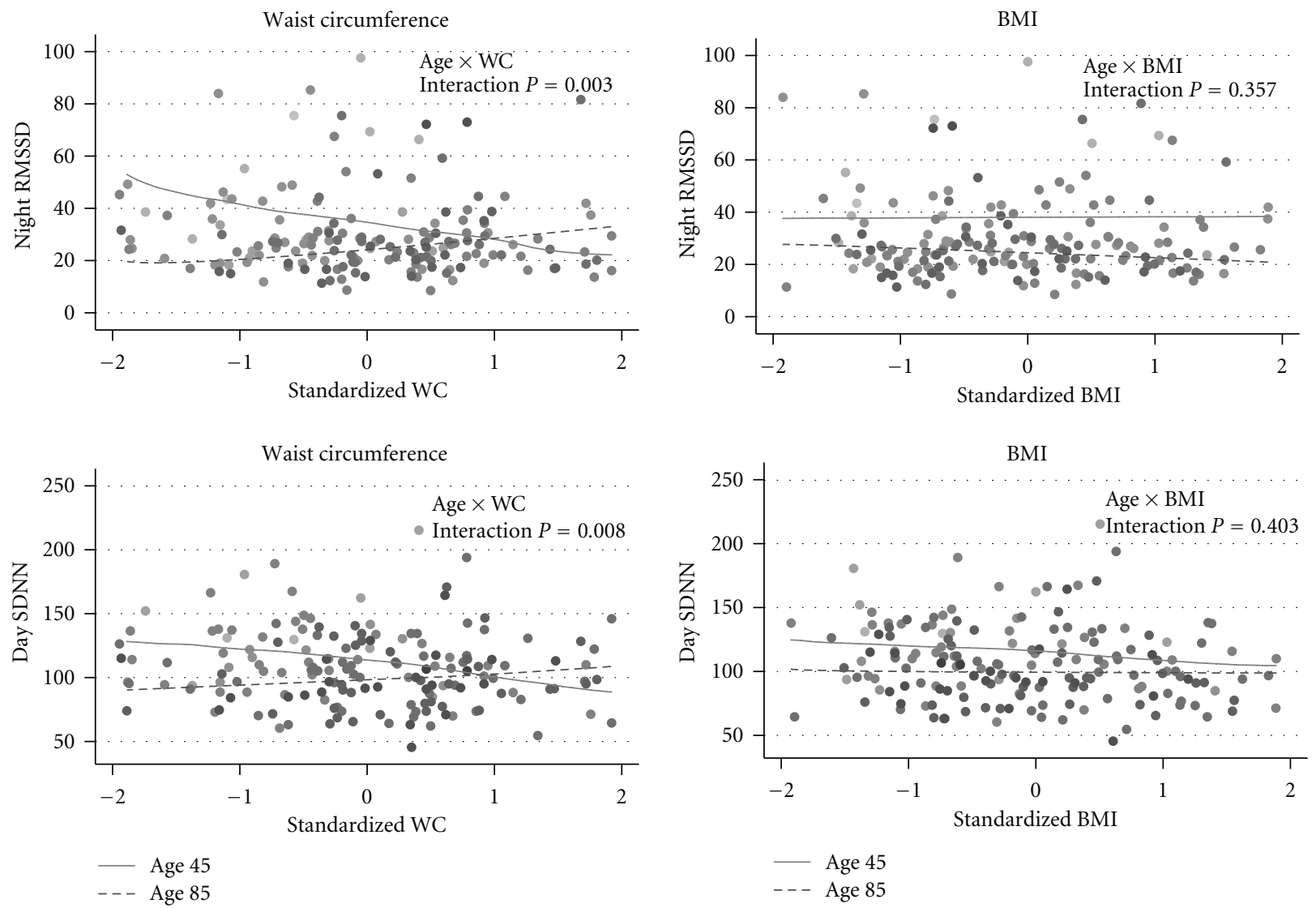

FIGURE 2: Data points are observed data; lines represent the predicted value of nighttime RMSSD (top row) and daytime SDNN (bottom row) (if the adiposity measure increased for an average participant at two ages: 45 years (solid lines) and 85 years (dashed lines)). Standardized waist circumference and standardized BMI are shown on $x$-axes to scale the measures similarly. The figures show differing relationships between waist circumference and HRV measures at young and old ages but not between BMI and HRV measures. Negative relationships were observed between waist circumference and RMSSD and SDNN in the 45 yo but no significant relationship in the 85 yo. Comparatively, the SDNN-BMI relationship is attenuated, compared to WC, for the 45-year olds and the RMSSD-BMI relationship is relatively flat at 45 and 85 years, with neither being statistically significant. ${ }^{*} x$-axes are standard deviations from mean waist circumference (women: $88 \mathrm{~cm}$, men: $98 \mathrm{~cm}$ ) or BMI $\left(27 \mathrm{~kg} / \mathrm{m}^{2}\right)$. ${ }^{*}$ Graded gray-scale data points from population illustrate increasing age (lighter is younger, darker is older, 26-96y). Models were adjusted for sex, race, OGTT glucose, blood pressure, and the interactions age-by-sex, age-by-race, the sexby-OGTT glucose. OGTT glucose $=2$-hour oral glucose tolerance test glucose result; RMSSD:root mean square of successive differences; SDNN:standard deviation of N-N intervals.

The relationship between central adiposity and ANS function is not a surprising finding. The ANS innervates fat depots, which are positively associated with catecholamine production by the ANS $[42,43]$. The biologic mechanisms whereby visceral fat could contribute to ANS dysfunction could involve adipocytokines secreted by fat cells [44, 45]. Although metabolic activity of central adipose tissue could account for the associations between ANS function and obesity, lack of association with BMI at older ages could be explained by the poor ability of BMI to accurately reflect body fat at older age [24]. This does not explain, however, the lack of associations observed in young persons. Future studies should examine the role of adipocytokines in mediating the effects of obesity on ANS function.

The lack of association for either measure of obesity at older ages supports recent arguments that "a little extra" around the middle may be good for older adults, or at least not as harmful as in young and middle age $[46,47]$.
Our findings lend some support for this or, at least, fail to demonstrate that central adiposity, within the range observed in this population, was associated with poorer ANS function in the oldest participants. In contrast, younger participants with more central adiposity appeared to have ANS function similar to older participants, that is, premature aging of the ANS.

The long-term sequelae of impaired ANS function manifested as lower HRV are unknown but could contribute to cardiovascular, lipid, and endocrine abnormalities as these mechanisms are regulated by the ANS. Evidence for this includes studies that demonstrate associations between lower HRV and coronary artery disease, heart failure, hyperlipidemia, hypertension, and prediabetic and diabetic states [9-11, 13, 48, 49]. Additionally, the relationship between central adiposity and ANS function observed in this study independent of blood pressure and glucose could explain how three of the most important components of 
TABLE 2: Relative risks (RR) percent change in SDNN and RMSSD for each standard deviation increase in the obesity variables: waist circumference (WC) and body mass index (BMI). Smaller Akaike's Information Criterion (AIC) indicates better fitted models.

\begin{tabular}{|c|c|c|}
\hline A. WC model & $\begin{array}{c}\text { Night RMSSD } \\
\text { RR } \\
(95 \% \text { CI }) P \text { value }\end{array}$ & $\begin{array}{c}\text { Day SDNN } \\
\text { RR } \\
(95 \% \text { CI }) P \text { value }\end{array}$ \\
\hline Age (per 10 year) & $\begin{array}{c}0.41 \\
(0.23,0.75) 0.004\end{array}$ & $\begin{array}{c}0.64 \\
(0.48,0.85), 0.002\end{array}$ \\
\hline Waist circumference (per $5 \mathrm{~cm}$ ) & $\begin{array}{c}0.73 \\
(0.58,0.92) 0.008\end{array}$ & $\begin{array}{c}0.85 \\
(0.76,0.95), 0.005\end{array}$ \\
\hline Age-by-waist circumference & $\begin{array}{c}1.05 \\
(1.01,1.08) 0.008\end{array}$ & $\begin{array}{c}1.02 \\
(1.01,1.04), 0.004\end{array}$ \\
\hline AIC & 819 & 953 \\
\hline B. BMI model & $\begin{array}{c}\text { Night RMSSD } \\
\text { RR } \\
\text { (95\% CI) } P \text { value }\end{array}$ & $\begin{array}{c}\text { Day SDNN } \\
\text { RR } \\
(95 \% \mathrm{CI}) P \text { value }\end{array}$ \\
\hline Age (per 10 year) & $\begin{array}{c}1.10 \\
(0.71,1.68), 0.673\end{array}$ & $\begin{array}{c}0.89 \\
(0.72,1.10), 0.300\end{array}$ \\
\hline Body mass index (BMI) & $\begin{array}{c}1.04 \\
(0.93,1.17), 0.496\end{array}$ & $\begin{array}{c}0.98 \\
(0.92,1.03), 0.404\end{array}$ \\
\hline Age-by-BMI & $\begin{array}{c}0.99 \\
(0.98,1.01), 0.403\end{array}$ & $\begin{array}{c}1.00 \\
(0.999,1.01), 0.428\end{array}$ \\
\hline AIC & 842 & 982 \\
\hline
\end{tabular}

Models adjusted for sex, race, blood pressure, oral glucose tolerance test (OGTT) 2-hour glucose, age-by-race, age-by-sex, OGTT 2-hour glucose-by-sex, and physical activity.

RR:relative risk;SDNN:standard deviation of normal-to-normal intervals.RMSSD:root mean square of successive differences in normal-to-normal intervals.

the metabolic syndrome (i.e., hypertension, diabetes, and obesity) act synergistically on the cardiovascular systemthat is, through the derangement of ANS function.

Regarding limitations, this study included a relatively small sample in a cross-sectional analysis. However, the BLSA is an ongoing study so future analyses will assess the robustness of these findings both cross-sectionally and longitudinally. Study participants were in relatively good health. Consequently, findings may not be generalizable to persons in poorer health. Nevertheless, observing the association between WC and HRV in healthy persons suggests that ANS dysfunction can be detected early and at relatively young ages, therefore providing an opportunity for early intervention. The cross-sectional design limits our ability to establish causal relationships, and, in fact, one hypothesis proposes that central effects of adipocytokines could alter sympathetic and parasympathetic balance, leading to changes in the distribution of visceral and subcutaneous fat [50]. Nevertheless, our findings provide important clues to guide future studies. For instance, longitudinal analyses will be conducted to determine how changes in WC and ANS function at younger ages contribute to health risk in late life.

In summary, we observed an inverse association between central adiposity measured by WC and ANS function at young and middle but not old-age. This was true for a measure of primarily parasympathetic function (RMSSD) as well as a measure of mixed sympathetic and parasympathetic function (SDNN). BMI was not associated with ANS function in this study. Future studies should examine whether metabolic activity of central adipose tissue explains these associations.

\section{Acknowledgment}

This study was sponsored by the National Institutes of Health and National Institute on Aging.

\section{References}

[1] C. J. Czura and K. J. Tracey, "Autonomic neural regulation of immunity," Journal of Internal Medicine, vol. 257, no. 2, pp. 156-166, 2005.

[2] A. C. Guyton and J. E. Hall, The Autonomic Nervous System. Textbook of Medical Physiology, WB Saunders, 10th edition, 1996.

[3] K. Nonogaki, "Obesity: autonomic circuits versus feeding," Nature Medicine, vol. 5, no. 7, pp. 742-743, 1999.

[4] M. Malik, "Heart rate variability: standards of measurement, physiological interpretation, and clinical use," Circulation, vol. 93, no. 5, pp. 1043-1065, 1996.

[5] H. Evrengul, H. Tanriverdi, S. Kose et al., "The relationship between heart rate recovery and heart rate variability in coronary artery disease," Annals of Noninvasive Electrocardiology, vol. 11, no. 2, pp. 154-162, 2006.

[6] R. E. Kleiger, P. K. Stein, and J. T. Bigger, "Heart rate variability: measurement and clinical utility," Annals of Noninvasive Electrocardiology, vol. 10, no. 1, pp. 88-101, 2005.

[7] M. T. La Rovere, G. D. Pinna, R. Maestri et al., "Short-term heart rate variability strongly predicts sudden cadiac death in 
chronic heart failure patients," Circulation, vol. 107, no. 4, pp. 565-570, 2003.

[8] D. Quilliot, F. Zannad, and O. Ziegler, "Impaired response of cardiac autonomic nervous system to glucose load in severe obesity," Metabolism, vol. 54, no. 7, pp. 966-974, 2005.

[9] E. B. Schroeder, L. E. Chambless, D. Liao et al., "Diabetes, glucose, insulin, and heart rate variability: the atherosclerosis risk in communities (aric) study," Diabetes Care, vol. 28, no. 3, pp. 668-674, 2005.

[10] J. P. Singh, M. G. Larson, C. J. O’Donnell et al., “Association of hyperglycemia with reduced heart rate variability (the framingham heart study)," The American Journal of Cardiology, vol. 86, no. 3, pp. 309-312, 2000.

[11] J. P. Singh, M. G. Larson, H. Tsuji, J. C. Evans, C. J. O’Donnell, and D. Levy, "Reduced heart rate variability and new-onset hypertension: insights into pathogenesis of hypertension: the framingham heart study," Hypertension, vol. 32, no. 2, pp. 293 297, 1998.

[12] D. Liao, J. Cai, R. W. Barnes et al., "Association of cardiac autonomic function and the development of hypertension. the aric study," American Journal of Hypertension, vol. 9, no. 12 I, pp. 1147-1156, 1996.

[13] J. F. Thayer and R. D. Lane, "The role of vagal function in the risk for cardiovascular disease and mortality," Biological Psychology, vol. 74, no. 2, pp. 224-242, 2007.

[14] C. Shibao, A. Gamboa, A. Diedrich et al., "Autonomic contribution to blood pressure and metabolism in obesity," Hypertension, vol. 49, no. 1, pp. 27-33, 2007.

[15] S. Lindmark, L. Lönn, U. Wiklund, M. Tufvesson, T. Olsson, and J. W. Eriksson, "Dysregulation of the autonomic nervous system can be a link between visceral adiposity and insulin resistance," Obesity Research, vol. 13, no. 4, pp. 717-728, 2005.

[16] R. H. Fagard, K. Pardaens, and J. A. Staessen, "Influence of demographic, anthropometric and lifestyle characteristics on heart rate and its variability in the population," Journal of Hypertension, vol. 17, no. 11, pp. 1589-1599, 1999.

[17] D. F. Dietrich, C. Schindler, J. Schwartz et al., "Heart rate variability in an ageing population and its association with lifestyle and cardiovascular risk factors: results of the sapaldia study," Europace, vol. 8, no. 7, pp. 521-529, 2006.

[18] B. Kuch, H. W. Hense, R. Sinnreich et al., "Determinants of short-period heart rate variability in the general population," Cardiology, vol. 95, no. 3, pp. 131-138, 2001.

[19] M. Vallejo, M. F. Márquez, V. H. Borja-Aburto, M. Cárdenas, and A. G. Hermosillo, "Age, body mass index, and menstrual cycle influence young women's heart rate variability: a multivariable analysis," Clinical Autonomic Research, vol. 15, no. 4, pp. 292-298, 2005.

[20] K. M. Flegal, M. D. Carroll, C. L. Ogden, and C. L. Johnson, "Prevalence and trends in obesity among us adults, 19992000," JAMA, vol. 288, no. 14, pp. 1723-1727, 2002.

[21] D. S. Freedman, D. F. Williamson, J. B. Croft, C. Baltew, and T. Byers, "Relation of body fat distribution to ischemic heart disease. The National Health and Nutrition Examination Survey I (HANES I)," American Journal of Epidemiology, vol. 142, pp. 53-63, 1995.

[22] W. B. Kannel, L. A. Cupples, R. Ramaswami, J. Stokes, B. E. Kreger, and M. Higgins, "Regional obesity and risk of cardiovascular disease; the framingham study," Journal of Clinical Epidemiology, vol. 44, no. 2, pp. 183-190, 1991.

[23] A. H. Kissebah, N. Vydelingum, and R. Murray, "Relation of body fat distribution to metabolic complications of obesity," The Journal of Clinical Endocrinology and Metabolism, vol. 54, no. 2, pp. 254-260, 1982.
[24] D. Gallagher, M. Visser, D. Sepúlveda, R. N. Pierson, T. Harris, and S. B. Heymsfieid, "How useful is body mass index for comparison of body fatness across age, sex, and ethnic groups?" American Journal of Epidemiology, vol. 143, no. 3, pp. 228-239, 1996.

[25] N. W. Shock, R. Greulich, and R. Andres, Normal Human Aging: The Baltimore Study of Aging, U. S. Department of Health and Human Services, Public Health, Washington, DC, USA, 2006.

[26] L. Adinolfi, A. Montano, B. Golia et al., "Electrocardiographic findings in a school-age population using a computerized system: calculation of standard norms for heart rate and pr, qrs, and qt intervals," Giornale Italiano Di Cardiologia, vol. 19, no. 2, pp. 145-152, 1989.

[27] L. M. Allan, S. R. J. Kerr, C. G. Ballard et al., "Autonomic function assessed by heart rate variability is normal in alzheimer's disease and vascular dementia," Dementia and Geriatric Cognitive Disorders, vol. 19, no. 2-3, pp. 140-144, 2005.

[28] J. Goldberger and A. Kadish, "Influence of sympathetic and parasympathetic maneuvers on heart rate variability," in Noninvasive Electrocardiology: Clinical Aspects of Holter Monitoring, A. Moss and S. Stern, Eds., pp. 207-223, WB Saunders, London, UK, 1995.

[29] C. J. Barrett, M. A. Navakatikyan, and S. C. Malpas, "Longterm control of renal blood flow: what is the role of the renal nerves?" American Journal of Physiology, vol. 280, no. 5, pp. R1534-R1545, 2001.

[30] H. Bonnemeier, U. K. H. Wiegand, A. Brandes et al., "Circadian profile of cardiac autonomic nervous modulation in healthy subjects: differing effects of aging and gender on heart rate variability," Journal of Cardiovascular Electrophysiology, vol. 14, no. 8, pp. 791-799, 2003.

[31] D. Goodman-Gruen and E. Barrett-Connor, "Sex differences in measures of body fat and body fat distribution in the elderly," American Journal of Epidemiology, vol. 143, no. 9, pp. 898-906, 1996.

[32] F. Iellamo, J. M. Legramante, M. Massaro, G. Raimondi, and A. Galante, "Effects of a residential exercise training on baroreflex sensitivity and heart rate variability in patients with coronary artery disease: a randomized, controlled study," Circulation, vol. 102, no. 21, pp. 2588-2592, 2000.

[33] American Diabetes A, "Role of blink reflex in diagnosis of subclinical cranial neuropathy in diabetic mellitus type II," Diagnosis and Classification of Diabetes Mellitus, vol. 29, supplement 1, pp. S43-S48, 2006.

[34] B. E. Ainsworth, W. L. Haskell, A. S. Leon et al., "Compendium of physical activities: classification of energy costs of human physical activities," Medicine and Science in Sports and Exercise, vol. 25, no. 1, pp. 71-80, 1993.

[35] S. N. Davis, C. Shavers, and F. Costa, "Differential gender responses to hypoglycemia are due to alterations in CNS drive and not glycemic thresholds," American Journal of Physiology, vol. 279, no. 5, pp. E1054-E1063, 2000.

[36] R. P. Hoffman, P. Vicini, W. I. Sivitz, and C. Cobelli, "Pubertal adolescent male-female differences in insulin sensitivity and glucose effectiveness determined by the one compartment minimal model," Pediatric Research, vol. 48, no. 3, pp. 384388,2000 .

[37] R. Lampert, J. R. Ickovics, C. J. Viscoli, R. I. Horwitz, and F. A. Lee, "Effects of propranolol on recovery of heart rate variability following acute myocardial infarction and relation to outcome in the beta-blocker heart attack trial," The 
American Journal of Cardiology, vol. 91, no. 2, pp. 137-142, 2003.

[38] D. D. Christou, P. P. Jones, A. E. Pimentel, and D. R. Seals, "Increased abdominal-to-peripheral fat distribution contributes to altered autonomic-circulatory control with human aging," American Journal of Physiology, vol. 287, no. 4, pp. H1530-H1537, 2004.

[39] M. Krotkiewski, P. Bjorntorp, L. Sjostrom, and U. Smith, "Impact of obesity on metabolism in men and women. importance of regional adipose tissue distribution," The Journal of Clinical Investigation, vol. 72, no. 3, pp. 1150-1162, 1983.

[40] K. M. Rexrode, V. J. Carey, C. H. Hennekens et al., "Abdominal adiposity and coronary heart disease in women," JAMA, vol. 280, no. 21, pp. 1843-1848, 1998.

[41] R. E. Van Pelt, E. M. Evans, K. B. Schechtman, A. A. Ehsani, and W. M. Kohrt, "Waist circumference vs body mass index for prediction of disease risk in postmenopausal women," International Journal of Obesity, vol. 25, no. 8, pp. 1183-1188, 2001.

[42] A. M. Dart, X. J. Du, and B. A. Kingwell, "Gender, sex hormones and autonomic nervous control of the cardiovascular system," Cardiovascular Research, vol. 53, no. 3, pp. 678-687, 2002.

[43] F. Kreier, E. Fliers, P. J. Voshol et al., "Selective parasympathetic innervation of subcutaneous and intra-abdominal fatfunctional implications," The Journal of Clinical Investigation, vol. 110, no. 9, pp. 1243-1250, 2002.

[44] G. Paolisso, D. Manzella, N. Montano, A. Gambardella, and M. Varricchio, "Plasma leptin concentrations and cardiac autonomic nervous system in healthy subjects with different body weights," The Journal of Clinical Endocrinology and Metabolism, vol. 85, no. 5, pp. 1810-1814, 2000.

[45] D. M. Penn, L. C. Jordan, E. W. Kelso, J. E. Davenport, and R. B. S. Harris, "Effects of central or peripheral leptin administration on norepinephrine turnover in defined fat depots," American Journal of Physiology, vol. 291, no. 6, pp. R1613-R1621, 2006.

[46] A. J. Campbell, G. F. S. Spears, J. S. Brown, W. J. Busby, and M. J. Borrie, "Anthropometric measurements as predictors of mortality in a community population aged 70 years and over," Age and Ageing, vol. 19, no. 2, pp. 131-135, 1990.

[47] D. H. Taylor and T. Østbye, "The effect of middle- and oldage body mass index on short-term mortality in older people," Journal of the American Geriatrics Society, vol. 49, no. 10, pp. 1319-1326, 2001.

[48] T. Kimura, T. Matsumoto, M. Akiyoshi et al., "Body fat and blood lipids in postmenopausal women are related to resting autonomic nervous system activity," European Journal of Applied Physiology, vol. 97, no. 5, pp. 542-547, 2006.

[49] D. Liao, J. Cai, W. D. Rosamond et al., "Cardiac autonomic function and incident coronary heart disease: a populationbased case-cohort study: the aric study," American Journal of Epidemiology, vol. 145, no. 8, pp. 696-706, 1997.

[50] E. Fliers, H. P. Sauerwein, J. A. Romijn et al., "Hiv-associated adipose redistribution syndrome as a selective autonomic neuropathy," The Lancet, vol. 362, no. 9397, pp. 1758-1760, 2003. 


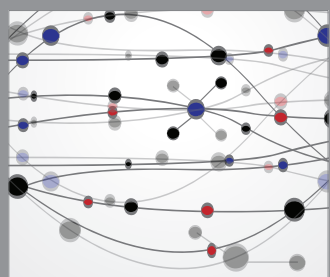

The Scientific World Journal
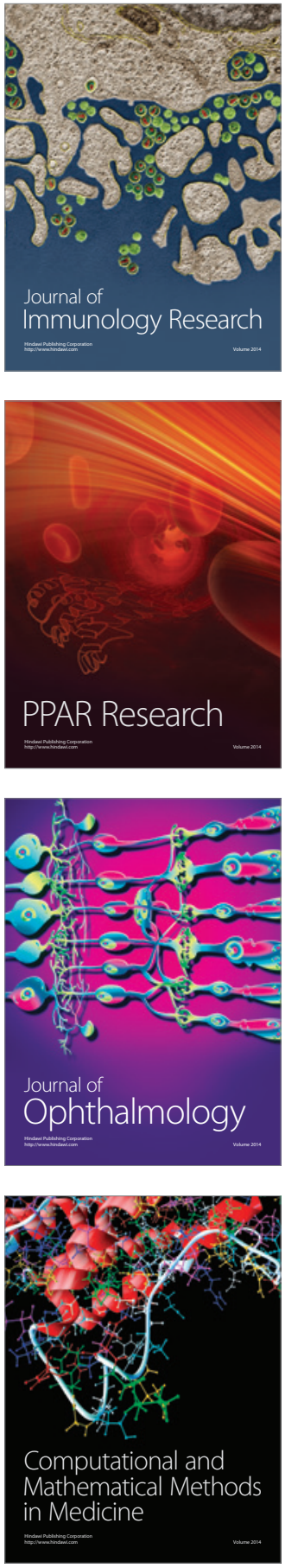

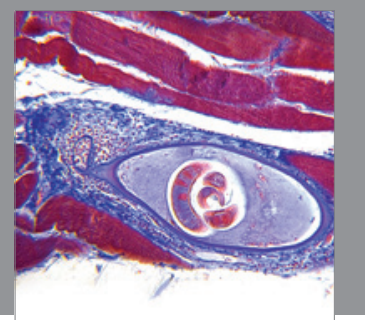

Gastroenterology

Research and Practice
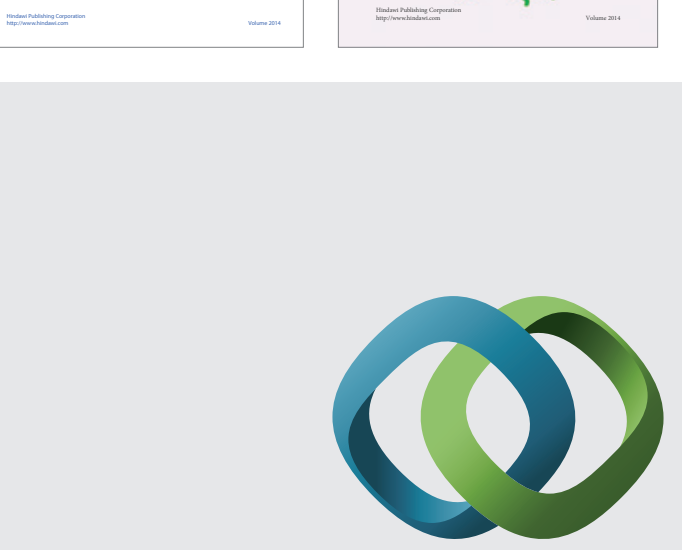

\section{Hindawi}

Submit your manuscripts at

http://www.hindawi.com
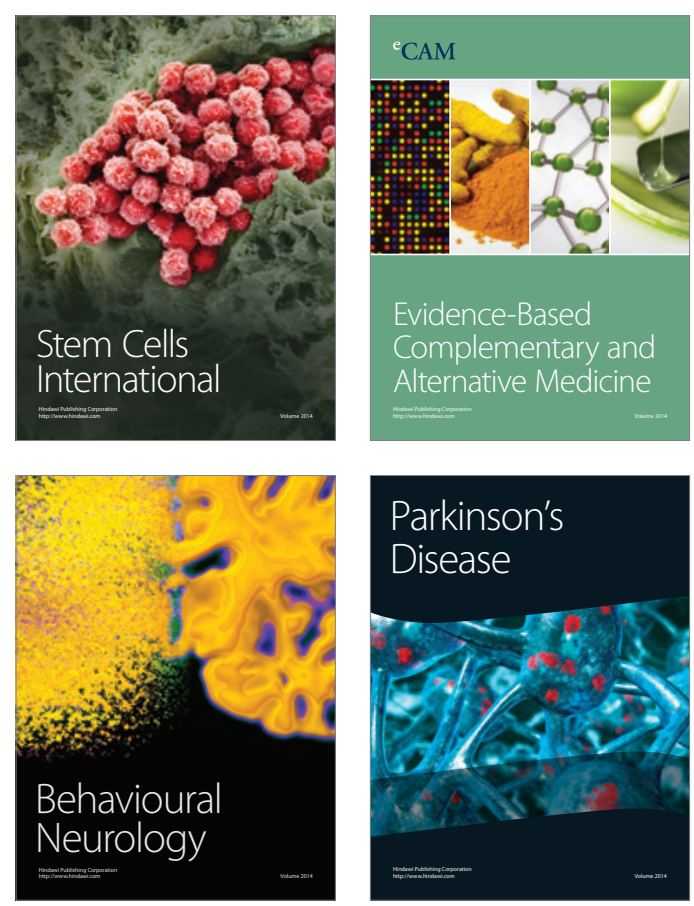

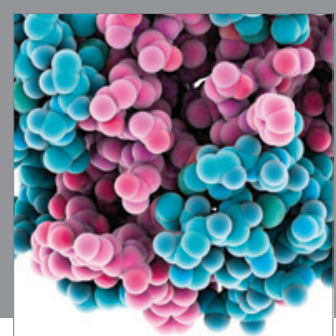

Journal of
Diabetes Research

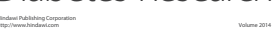

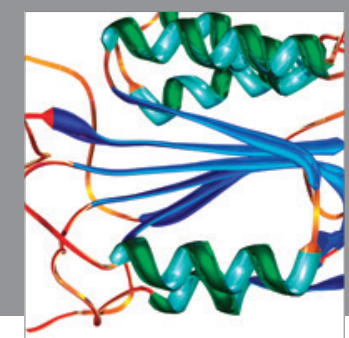

Disease Markers
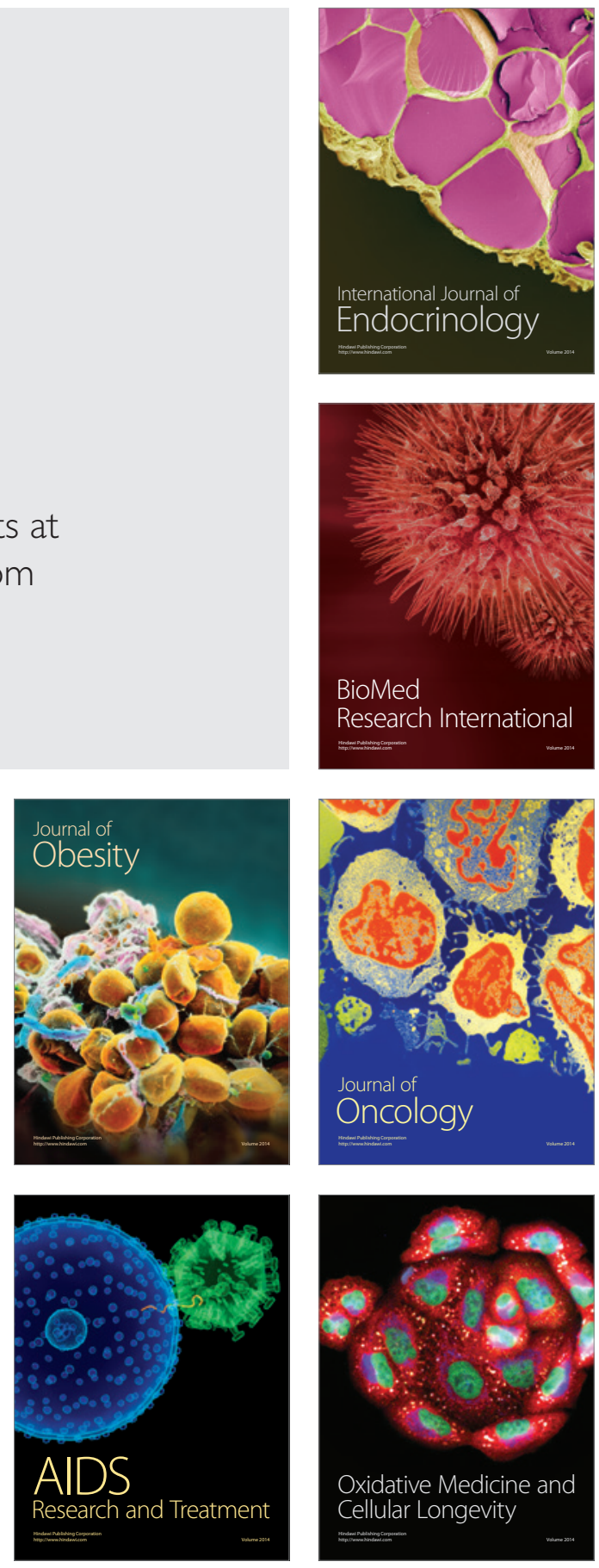